\title{
Hepatitis C and sex
}

\author{
Authors: Emma E Page ${ }^{\mathrm{A}}$ and Mark Nelson ${ }^{\mathrm{B}}$
}

An outbreak of acute hepatitis C among HIV-positive men who have sex with men (MSM) in the last decade has been shown to be sexually transmitted. Initially recreational drug use, in particular drug injection, was not prevalent among those becoming infected with hepatitis $\mathrm{C}$. However more recently chemsex (the use of drugs to enhance sexual experience) and its associated drugs, which are not uncommonly injected, have become more frequently reported among those diagnosed with hepatitis $\mathrm{C}$. It is hoped that the widespread introduction of direct-acting antivirals and upscaling of numbers treated may have a positive impact on this epidemic. However their introduction may negatively impact on the perceived risk of acquiring hepatitis $C$ and in conjunction with the introduction of HIV transmission prevention strategies may result in increased transmissions and spread to the HIVnegative MSM population.

\section{Introduction}

Historically hepatitis C virus (HCV), unlike other bloodborne viruses such as hepatitis B virus (HBV) and human immunodeficiency virus (HIV), has not been viewed as a sexually transmitted infection (STI). However an outbreak of acute hepatitis $\mathrm{C}$ (AHC) among HIV-positive men who have sex with men (MSM) in the last decade has led HIV and sexual health physicians to question this. It is now well accepted, though maybe less widely known, that HCV is a STI. It is not as readily transmitted sexually as HBV or HIV but given the right environment will transmit. To date this STI has almost exclusively been seen among MSM the majority of who are HIV positive. This cohort tends to be different from the classic intravenous drug user (IDU) population with higher indices of social stability such as employment, home ownership and history of imprisonment. ${ }^{1}$ For many, a diagnosis of HCV brings back memories of their diagnosis with HIV with similar fears and stigmas both from the general population and also from within the MSM community. However a more positive parallel with HIV is the recent explosion of new direct-acting antivirals (DAAs) which offer the vast majority viral clearance;

Authors: ${ }^{A}$ locum consultant GUM and virology, Departments of Genitourinary Medicine and Virology, Leeds Teaching Hospitals Trust, Leeds, UK; ${ }^{\mathrm{B}}$ consultant HIV physician, Department of Sexual Health and HIV, Chelsea and Westminster Hospital, London, UK something many may not have achieved under the 'old' treatment regimens of dual therapy with pegylated interferon (IFN) and ribavirin (RBV) given their poor efficacy in HIV/ $\mathrm{HCV}$ co-infection. The introduction of more effective therapies is not the only factor likely to affect the future of HCV in HIVpositive individuals. The evolution of chemsex, with the most common drugs (crystal methamphetamine and mephedrone) now frequently being injected, the widespread introduction of treatment as prevention (TasP), pre-exposure prophylaxis (PrEP) and imminent scale-up of HIV therapy to all are likely to impact.

\section{The start of the epidemic}

The earliest reports of an outbreak of AHC in HIV-positive MSM emerged from Europe; London, Paris and Berlin. ${ }^{2-4}$ In the UK in 2008 the Health Protection Agency set up an enhanced surveillance of recently acquired HCV. ${ }^{5}$ Data were collected prospectively (January 2008 - March 2010) from 22 UK sites in London and the south-east. 218 episodes of recently acquired HCV were identified: $84 \%$ were in London, $63 \%$ were UK born and $90 \%$ were of white ethnicity. The majority (94\%) were HIV positive. Only $21 \%$ admitted to IDU, while other recreational drug use was common. High-risk sexual practices were common, with the majority admitting to unprotected anal intercourse (UPAI), one-third to fisting and two-thirds being diagnosed with a concomitant STI (most commonly syphilis). These findings were common to outbreaks in Europe, America and Australia. ${ }^{6-8}$ Thus a debate on the possibility of sexual transmission of HCV was opened.

\section{Evidence for sexual transmission}

Sexual transmission occurs when infected bodily fluids or blood are exchanged across mucosal surfaces. It is generally accepted that HCV RNA can be demonstrated in semen although it is not present consistently (10-30\% of HCV infected men) and titres tend to be low. ${ }^{9}$ Prospective studies have shown that sexual transmission among heterosexual couples is rare. ${ }^{10,11}$ Until recently sexual transmission was not felt to be any more frequent among MSM with studies such as the Omega Cohort Study demonstrating an incidence rate of 0 per 1,000 patient-years. $^{12}$

Despite this lack of evidence for sexual transmission of HCV there were reasons to suspect its involvement in the increased incidence of HCV among HIV-positive MSM. First, several studies have demonstrated the increased incidence of HCV in 
HIV-positive MSM compared to HIV-negative MSM. ${ }^{13,14}$ HIV may enhance the transmission and acquisition of HCV in a number of ways. HCV RNA is found more frequently in the semen of HIV-positive men (37.8\%) than in those with HCV mono-infection (18.4\%), and at higher levels. ${ }^{15,16}$ In addition rectal mucosal viral susceptibility is likely to be enhanced in HIV disease due to the well-documented detrimental effects of HIV infection on gastrointestinal mucosal barrier function and the gastrointestinal-associated lymphoid tissue. ${ }^{17}$ Second, a significant number of HIV-positive MSM being diagnosed with AHC had concurrent ulcerative STIs. This not only gives evidence for sexual risk, but mucosal barrier breakdown would enhance the transmission of any opportunistic infection. Third, the availability of highly active antiretroviral therapy (HAART) has been followed by a decrease in the perceived threat of HIV leading to: increased rates of UPAI with casual partners (15.3\% in 1998 to $38.8 \%$ in 2001$),{ }^{18}$ more prevalent serosorting (identifying other HIV-positive men with whom one can 'safely' have condom-less sex $)^{18}$ and increased rates of STIs. ${ }^{19}$ Finally the precedent had already been set with the emergence of LGV demonstrating the susceptibility of this network to the introduction and spread of new opportunistic STIs. ${ }^{20}$

Danta et al provided the initial evidence that the recent epidemic was being driven by sexual transmission. ${ }^{21} 111$ HIV-positive MSM diagnosed with AHC between 1999 and 2005 were recruited to a phylogenetic and case-control study. Phylogenetic trees revealed multiple independent monophyletic clusters, which contained sequences from multiple sites. This suggested the epidemic was being driven by behavioural or environmental factors as opposed to viral change, and that transmission was occurring within close social networks. Molecular clock data gave evidence for increased transmission since 1995 (the year HAART was introduced). In multivariate analysis drug use was not associated with the acquisition of $\mathrm{HCV}$, while group sex involving insertive or receptive UPAI and insertive or receptive fisting were the only independent predictors of the acquisition of $\mathrm{HCV}$.

\section{Key points}

Hepatitis C virus (HCV) can be sexually transmitted within the men who have sex with men (MSM) population.

Chemsex (the use of drugs to enhance sexual experience) is prevalent within pockets of the MSM community.

Injecting of chemsex drugs is becoming more frequent.

Direct-acting antivirals (DAAs) may lead to a reduction in the prevalence of HCV in the HIV-positive population if there is significant scale up of numbers treated

DAAs may alter lifestyle behaviours with increased risk taking and spread of the epidemic of acute hepatitis C to the HIVnegative MSM population

KEYWORDS: HIV, hepatitis $\mathrm{C}$, men who have sex with men, sexual transmission, chemsex

\section{The impact of chemsex}

Chemsex is a term used to describe the use of drugs to enhance sexual experience and has been most commonly associated with the MSM community. The use of illicit drugs or legal highs is not new on the gay club scene where, for years, drug use has been normalised. However recently there has been an evolution in the drugs used with a move away from the classic 'club drugs' ecstasy, cocaine and ketamine to the replacement of these with more dangerous and more addictive drugs like crystal methamphetamine, gammahydroxybutrate (GHB) and mephedrone. These are stimulant drugs that increase heart rate and blood pressure; in addition they are known to trigger feelings of euphoria and sexual arousal. These drugs can facilitate long sexual sessions with multiple partners that can extend over several days. The rise in reported rates of slamming (injecting) and the consequential traumatic sexual practices associated with chemsex only enhance the transmission risks of HCV. Data from Antidote (a London-based drugs and alcohol support service for the LGBT community) demonstrate this change in drug use with the main presentation in 2004/5 being alcohol use and the most common drugs cocaine, ecstasy and ketamine, with only $2 \%$ of service users reporting GHB use and none reporting crystal methamphetamine. Data on mephedrone were not even being collected at this time. ${ }^{22}$ By 2013/14 51\% were using crystal methamphetamine, 46\% GHB and $64 \%$ mephedrone. The vast majority using these drugs are doing so in sexualised situations with rates of reported injecting increasing from $0 \% 2004 / 5$ to $49 \%$ in $2013 / 14$. The choice of venue has also moved from clubs into private parties, with many social apps such a 'Grindr' and 'BarebackRT' facilitating relative anonymity. This increase in chemsex led to the London Boroughs of Lambeth, Southwark and Lewisham commissioning the Chemsex Study. ${ }^{23}$ It demonstrated that onequarter (all of whom were HIV positive) of those taking part in chemsex planned to engage in UPAI and one-third found it difficult to prevent engagement in STI transmission risk behaviour, which they subsequently regretted. Use of chemsex drugs was shown to be much more prevalent in these boroughs than the rest of London and more prevalent in London than the rest of the UK. However it would be misleading to suggest that chemsex and increased injecting of illicit drugs was not an issue for pockets of MSM throughout the country.

\section{Future of hepatitis c and sex}

With the advent of DAAs the future of hepatitis $\mathrm{C}$ is changing. As highly effective IFN-free therapeutic options are rolled out among the HIV/HCV co-infected population there is likely to be an impact on the current epidemic of AHC in HIV-positive MSM. It is possible that increased treatment uptake will lead to a reduction in the infective pool and reduced rates of transmission. Modelling work to examine the impact of DAAs on the prevalence of HCV has shown that switching to IFN-free therapy alone is unlikely to impact, however if this is combined with a significant scale up of numbers treated it is likely to have a real impact on the prevalence of chronic hepatitis $\mathrm{C}$ over time. $^{24}$

However as has been demonstrated in HIV and gonorrhoea epidemics, you cannot control an epidemic without controlling the core transmission group. ${ }^{25}$ During the era of IFN, high 
Table 1. Current and future studies with DAAs in acute HCV. Reproduced with permission. ${ }^{28}$

\begin{tabular}{|c|c|c|c|c|c|}
\hline Study name & Coordinator & DAAs & HCV genotype & Duration, weeks & HIV status \\
\hline DAHHS & Erasmus MC & $\mathrm{BOC}+$ pegIFN + RBV & 1 & 12 & Pos \\
\hline CHAT & UKB & TPV + pegIFN + RBV & 1 & 12 & Pos \\
\hline DARE-C I & Kirby Institute & TPV + pegIFN + RBV & 1 & $8-24$ & $\mathrm{Neg}+$ pos \\
\hline DARE-C II & Kirby Institute & $\mathrm{SOF}+\mathrm{RBV}$ & All & 6 & $\mathrm{Neg}+$ pos \\
\hline SWIFT-C & ACTG & $\mathrm{SOF}+\mathrm{RBV}$ & All & 8 vs 12 & Pos \\
\hline SOL & UKB & SOF + LDV & 1,4 & 6 & Pos \\
\hline Hep-Net Acute HCV & $\mathrm{MHH}$ & SOF + LDV & 1 & 6 & Neg \\
\hline
\end{tabular}

rates of reinfection with AHC in HIV-positive MSM have already been well documented. ${ }^{26}$ The widespread introduction of IFN-free therapies may impact on an individual's riskbenefit assessment with a reduced 'fear' of contracting AHC. A resultant shift in an individual's lifestyle behaviours has the potential not only to increase rates of transmission but also the pool of at-risk individuals. First, as discussed above, chemsex and injecting are becoming more prevalent in the MSM community and wherever there is the potential for parenteral transmission, rates of HCV transmission will increase. Second, there is the potential risk of the spread of this epidemic into the HIV-negative MSM population. Until now this epidemic has been ring fenced in the HIV-positive MSM population for a number of reasons. Firstly it is well known that HIV is more readily sexually transmitted than HCV. HIV-negative individuals involved in high-risk sexual networks are likely to contract HIV initially. Infection with HIV is likely to facilitate HCV transmission via any route and ongoing highrisk practices will increase their chances of contracting AHC. Secondly serosorting has effectively contained this epidemic within the HIV-positive population. However with the widespread introduction of PrEP (ClinicalTrials.gov Identifier: NCT02065986) and TasP ${ }^{27}$ as HIV transmission prevention strategies, this may change. These strategies may well reduce HIV incidence, but as HIV-positive and -negative MSM come to understand and appreciate their impact we may well see a loss of serosorting with increasing numbers of HIV-negative MSM exposed to the pool of HIV-positive HCV-infected MSM. Data from the UK suggest we are already beginning to see spread to the HIV-negative MSM population. ${ }^{28}$

Finally a major issue facing policy decision-makers at present is the cost of the new DAAs, with a 12-week treatment course costing in the region of $\mathfrak{E} 35,000$. In the UK over the last 18 months we have seen a surge in funding, second only to cancer drug funding, for the new DAAs for chronic hepatitis C, with those with cirrhosis being prioritised. Treatment of AHC is not mentioned in these documents largely because of a lack of data (although there are studies underway (Table 1)), let alone costeffectiveness data. Thus at present individuals with AHC can either opt for treatment with RBV and IFN for 24-48 weeks or wait for 6 months until they are classed as chronic when, from February 2016, they will be eligible for just 8 weeks of a singletablet regimen via NICE guidance (TA363). At these costs the need to re-treat individuals re-infected with AHC will lead to difficult ethical and public health decisions.

\section{References}

1 Mattews GV, Pham ST, Hellard M et al. Patterns and characteristics of hepatitis $\mathrm{C}$ tramsission clusters among HIV-positive and HIVnegative individuals in the Australian Trial in Acute Hepatitis C. Clin Infect Dis 2011;52:803-11.

2 Browne R, ASboe D, Gileece Y et al. Increased numbers of acute hepatitis $\mathrm{C}$ infections in HIV positive homosexual men; is sexual transmission feeding the increase? Sex Transm Infect 2004;80:326-7.

3 Cambotti L, Batisse D, Colin-de Verdiere $\mathrm{N}$ et al. Acute hepatitis C collaborating group. Acute hepatitis $\mathrm{C}$ infection in HIV positive men who have sex with men in Paris, France 2001-2004. Euro Surveill 2006;10:115-7.

4 Vogel M, Bleniek B, Jessen $\mathrm{H}$ et al. Treatment of acute hepatitis C infection in HIV-infected patients: a retrospective analysis of eleven cases. J Viral Hepat 2006;12:207-11.

5 Health Protection Agency. Hepatitis C in the UK: 2011 Report. London: HPA, 2011.

6 Gotz HM, van Doornum G, Niesters HGM et al. A cluster of acute hepatitis $C$ virus infection among men who have sex with men results from contact tracing and public health implications. AIDS 2005;19:969-74.

7 Matthews GV, Hellard M, Kaldor J et al. Further evidence of HCV sexual transmission among HIV-positive men who have sex with men: response to Danta et al. AIDS 2007;21:2112-3.

8 Luetkemeyer A, Hare CB, Stansell J et al. Clinical presentation and course of acute hepatitis C infection in HIV-infected patients. J Acquir Immune Defic Syndr 2006;41:31-6.

9 Leruez-Ville M, Kunstmann JM, De Almeida M et al. Detection of HCV in the semen of infected men. Lancet 2000;356:42-3.

10 Marincovich B, Castilla J, del Romero J et al. Low incidence of hepatitis $\mathrm{C}$ virus transmission in a prospecitive study. J Gastroenterol Hepatol 2000;15:391-5.

11 Vandelli C, Renzo F, Romano L et al. Lack of evidence of sexual transmission of hepatitis $\mathrm{C}$ amongst monogamous couples: results of a 10-year prospective follow-up study. Am J Gastroentrerol 2004;99:855-9.

12 Alary M, Joly J, Vincelette J et al. Lack of evidence of sexual transmission of hepatitis $\mathrm{C}$ virus in a prospective cohort study of men who have sex with men. Am J Public Health 2005;95:502-5.

13 van de Laar T, Van De Bij A, Prins M et al. Increase in HCV incidence among men who have sex with men in Amsterdam most likely caused by sexual transmission. J Infect Dis 2007;196:230-8.

14 Richardson D, Fisher M, Sabin C et al. Sexual transmission of hepatitis in MSM may not be confined to those with HIV infection. J Infect Dis 2008;197:1213-4.

15 Briat A, Dulioust E, Galimand J et al. Hepatitis C virus in the semen of men co-infected with HIV-1: prevalence and origin. AIDS 2005; 19:1827-35. 
16 Pasquier C, Bujan L, Daudin M et al. Intermittent detection of hepatitis $\mathrm{C}$ virus (HCV) in semen form men with human immunodeficiency virus type 1 (HIV-1) and HCV. J Med Virol 2003;69:344-9.

17 Brenchly JM, Schaker TW, Ruff et al. CD4+ T cell depletion during all stages of HIV disease occurs predominantly in the gastrointestinal tract. J Exp Med 2004;200:749-59.

18 Elford J, Bolding G, Sherr L. High risk sexual behavior increases among gay men between 1998 and 2001: what is the role of HIV optimism? AIDS 2002;16:1537-44.

19 Ostrow DE, Fox KJ, Chmiel JS et al. Attitudes towards HAART are associated with sexual risk taking among HIV infected and uninfected homosexual men. AIDS 2002;16:775-80.

20 Ward H, Alesander S, Carder C et al. The prevalence of lymphogranuloma venereum infection in men who have sex with men: results of a multicentre case finding study. Sex Transm Infect 2009;85:173-5.

21 Danta M, Brown D, Bhangani S et al. Recent epidemic of acute hepatitis $\mathrm{C}$ virus in HIV-positive men who have sex with men linked to high-risk sexual behaviors. AIDS 2007;21:983-91.

22 Moncrieff M. Out of your mind. London Friend, 2 June 2014.

23 Martin NK, Foster GR, Vilar J et al. HCV treatment rates and sustained viral responses among people who inject drugs in seven UK sites: real world results and modeling of treatment impact. J Viral Hepat 2015:22;399-408.

24 Hethcote HW, Yorke J. Gonorrhoea transmission dynamics and control. New York: Springer-Verlag, 1974.

25 Martin T, Martin N, Hickman M et al. Hepatitis C virus reinfection incidence and treatment outcome among HIV-positive MSM. AIDS 2013:27:2551-7.

26 Rodger A, Brunn T, Cambiano V et al. No one with undetectable viral load transmits HIV in PARTNER study. 21st Conference on Retroviruses and Opportunistic Infections, March 3-6, 2014; abstract 153LB.

27 McFaul K, Maghlaoui A, Nzuruba M et al. Acute hepatitis C infection in HIV-negative men who have sex with men. J Viral Hep 2015:22;535-8.

28 Boesecke C, Page E. Direct-acting antiviral-based therapy for acute hepatitis C coinfection. Curr Opin HIV AIDS 2015;10:330-6.

Address for correspondence: Dr EE Page, Virology,

Microbiology Department, Old Medical School, Leeds General Infirmary, Leeds LS1 3EX, UK.

Email: emma.page7@nhs.net
Assessing trainees in the workplace An e-learning module for secondary care doctors

Three hours of CPD-approved interactive learning covering:

$>$ feedback

$>$ supervised learning events (SLEs)

$>$ workplace-based assessments (WPBAs)

$>$ the role of the Annual Review of Competence Progression (ARCP).

For more information please visit: www.rcplondon.ac.uk/elearning

\section{Royal College} of Physicians

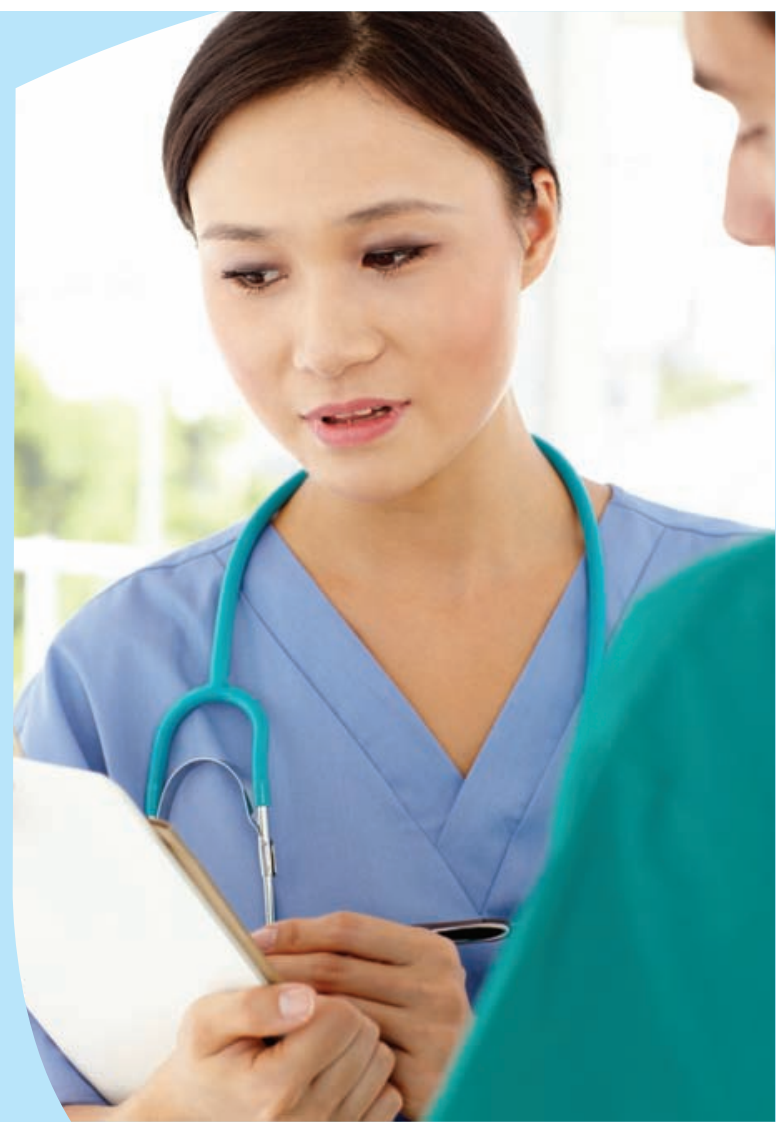

